\title{
LA EDUCACIÓN INCLUSIVA EN EL MARCO DE LA EDUCACIÓN SUPERIOR
}

EDUCAÇÃO INCLUSIVA NO ÂMBITO DA EDUCAÇÃO SUPERIOR

INCLUSIVE EDUCATION WITHIN THE FRAMEWORK OF HIGHER EDUCATION

\author{
Gloria Elena Landero JÁCOME ${ }^{1}$ \\ María Cristina Miranda ÁLVAREZ ${ }^{2}$
}

\begin{abstract}
RESUMEN: El presente artículo muestra a la educación inclusiva como aquella que permite la construcción de dimensiones que promueven la equidad, la colaboración y el desarrollo en la comunidad universitaria de nuevas miradas hacia aquellos grupos tradicionalmente excluidos. Primero se revisa el concepto de educación inclusiva, reconociendo que aunque se han hecho progresos considerables en este tema, aún quedan algunos aspectos importantes que permanecen todavía sin resolver, en segundo lugar se advierte la necesidad de identificar algunos vacíos teóricos y procedimentales que plantean retos fundamentales para la formación docente del nivel superior y en tercer lugar se identifican aquellas estrategias que promueven la construcción de espacios incluyentes que buscan minimizar o reducir las barreras para la presencia, la participación y el aprendizaje de los estudiantes.
\end{abstract}

PALABRAS CLAVE: Inclusión educativa. Formación docente. Educación superior.

RESUMO: Este artigo considera educação inclusiva aquela que permite a construção de dimensões que promovam a equidade, a colaboração e desenvolvam novas perspectivas na comunidade universitária em relação aos grupos tradicionalmente excluídos. Primeiro, o conceito de educação inclusiva é revisado, reconhecendo que, embora tenham sido feitos progressos consideráveis nessa área, ainda existem aspectos importantes que não foram resolvidos; segundo, reconhece-se a necessidade de identificar lacunas teóricas e processuais que levantam desafios fundamentais à formação de professores de nível superior; e, em terceiro lugar, são identificadas estratégias que promovam a construção de espaços inclusivos que busquem minimizar ou reduzir barreiras à presença, participação e aprendizado dos alunos.

PALAVRAS-CHAVE: Inclusão educacional. Formação de profesores. Educação superior.

ABSTRACT: This paper shows inclusive education as that which allows the construction of dimensions that promote equity, collaboration and development in the university community of new looks towards those groups traditionally excluded. Firstly, the concept of inclusive education is reviewed, recognizing that although considerable progress has been made in this

\footnotetext{
${ }^{1}$ Universidad Veracruzana (UV), Veracruz - Mexico. Secretaria de la Facultad de Pedagogía. Maestría en psicoterapia infantil Guestalt y Doctorado en Educación. ORCID: https://orcid.org/0000-0002-4334-7173. E-mail: gllandero@uv.mx

${ }^{2}$ Universidad Veracruzana (UV), Veracruz - Mexico. Profesor de Tiempo Completo en Facultad de Pedagogía. Doctorado en Educación (ELCPAPO) - Mexico. ORCID: https://orcid.org/0000-0002-1993-1038. E-mail: cmiranda@uv.mx
}

RIAEE - Revista Ibero-Americana de Estudos em Educação, Araraquara, v. 15, n. esp. 4, p. 2713-2726, dez., 2020. e-ISSN: 1982-5587 
area, there are still some important aspects that remain unresolved. Secondly, it is necessary to identify some theoretical and procedural gaps that pose fundamental challenges for teacher training at the higher level, and thirdly, it identifies those strategies that promote the construction of inclusive spaces that seek to minimize or reduce barriers to the presence, participation and learning of students.

KEYWORDS: Educational inclusion. Teacher education. Higher education.

\section{Inclusión educativa}

Una gran parte de las ideas que dieron nacimiento a la educación inclusiva, emanan de una serie de principios y exigencias de organismos internacionales, surgieron bajo la protección de la Declaración Universal de los Derechos Humanos en 1948, principalmente en el artículo 26, que señala: “Toda persona tiene derecho a la educación. La educación se dirigirá al pleno desarrollo de la personalidad humana y a fortalecer el respeto a los derechos humanos y a las libertades fundamentales". Por su parte la UNESCO (2005) define el término de Inclusión educativa, desde el contexto de aquél proceso que identifica y responde "a la diversidad de las necesidades de todos los estudiantes a través de la mayor participación en el aprendizaje, las culturas y las comunidades, y reduciendo la exclusión en la educación" (9), entre otros factores, cabe destacar, que la educación inclusiva no se refiere a cómo se educa a un grupo especial de alumnos, sino a cómo se educa a todos (TOBÓN, 2012).

En general, estos conceptos ponen en relieve la necesidad de identificar a la educación inclusiva como aquella que busca la participación de todos los estudiantes sin importar las características particulares que estos posean, siendo ellos los principales responsables de su proceso de aprendizaje, en vista de que tienen que interactuar con pares para intercambiar conocimientos, teniendo en cuenta el contexto para poder resolver diferentes problemas o situaciones (GÓMEZ; GARCÍA, 2017). El autor Salinas (2013), también reconoce a la inclusión con referencia a "un proceso orientado a responder a la diversidad de necesidades de todos los estudiantes, con el objetivo de incrementar su participación en el aprendizaje y eliminar la exclusión en y desde la educación” (80).

Según el punto de vista de Ainscow (2006), reconoce la concurrencia de tres elementos en el concepto de inclusión: presencia, aprendizaje y participación, al que considera un proceso de mejora sistemático que deben afrontar las administraciones educativas y los centros escolares; para intentar eliminar las barreras de distinto tipo que limitan la presencia, el aprendizaje y la participación del alumno en las instituciones en las que están escolarizados.

RIAEE - Revista Ibero-Americana de Estudos em Educação, Araraquara, v. 15, n. esp. 4, p. 2713-2726, dez., 2020. e-ISSN: 1982-5587 
En vista de todas las concepciones que en relación a la educación inclusiva se han expuesto hasta ahora, se pone en relieve la necesidad de analizar su importancia en el contexto escolar, reconociendo que en el caso de México, se le ha dado gran relevancia sobre todo en la educación del nivel básico, esta concepción parece respaldada por los programas de educación inclusiva que ha propuesto la Secretaría de Educación Pública (SEP), entre ellos se encuentra la iniciativa denominada Estrategia de equidad e inclusión en la educación básica: para alumnos con discapacidad, aptitudes sobresalientes y dificultades severas de aprendizaje, conducta o comunicación, la cual se inserta en el contexto del planteamiento pedagógico del Modelo Educativo, en esencia, se identifica bajo el concepto de "esfuerzo sin precedentes que se inscribe en el marco de una estrategia transversal, la cual busca conformar una educación inclusiva sustentada en la equidad, la justicia y la igualdad como elementos indispensables para asegurar la calidad en los servicios educativos" (SEP, 2018, p. 4).

Sin embargo, algunas investigaciones señalan que actualmente se cuenta con un sistema educativo diversificado, en donde aún existen varias dificultades estructurales, que obstaculizan la manifestación de todo el potencial de la educación inclusiva, por lo tanto, se tiene el reto de resolver estos problemas desde una perspectiva integral, sostenible y sistémica, para así atender y satisfacer a todos los niveles sociales y económicos. Por su parte Fernández (2017), señala que entre otros aspectos, se hace necesario rediseñar el sistema institucional para abarcar temas de equidad e inclusión.

Dentro del marco legal en México, desde 2011 existe la Ley General para la Inclusión de las Personas con Discapacidad, con una última reforma en 2018; donde se establece que el "Estado deberá promover, proteger y asegurar el pleno ejercicio de los derechos humanos y libertades fundamentales de las personas con discapacidad, asegurando su plena inclusión a la sociedad en un marco de respeto, igualdad y equiparación de oportunidades.” (p. 1).

El concepto de inclusión que analizan Ainscow y Miles (2008) es particularmente interesante; sobre todo porque nos presenta diversas perspectivas de la misma, por una parte, señalan que la inclusión tiene relación con la discapacidad y las necesidades educativas especiales, en otro aspecto se identifica como una respuesta a las exclusiones disciplinarias, también se ubica orientada a todos los grupos vulnerables a la exclusión y por último con disposición para la promoción de una escuela y una educación para todos.

\section{La inclusión en la educación superior}

A partir de los comentarios precedentes, son particularmente interesantes los resultados obtenidos por Romero y García (2013) y García (2011), los cuales concluyen que se han llevado 
a cabo muchos esfuerzos por incorporar la educación inclusiva en las escuelas de formación básica. En el caso de México, se han realizado iniciativas importantes en relación con la formación docente y la incorporación de niños con discapacidad a escuelas regulares, al respecto, Ocaña, Hernández, Sánchez, Linares, Guerra, Reyes, Soto (2019), señalan, que a pesar de que México cuenta con la Ley General de Educación en materia de educación inclusiva, desde el año 2016, ésta solo aplica para la educación básica general. En esas circunstancias, la educación superior ha quedado como una asignatura aún pendiente de integrar procesos alineados con el Modelo Educativo de la SEP (Secretaria de Educación Pública) que propone la formación integral de los estudiantes en la convicción y capacidades necesarias para contribuir a la construcción de una sociedad más justa e incluyente, respetuosa de la diversidad, atenta y responsable hacia el interés general (SEP, 2016). En concordancia con los planteamientos de la SEP, se espera la configuración de una educación universitaria que contemple en sus procesos, una visión incluyente. Cabe señalar que el término incluyente, tiene diversas connotaciones, de acuerdo con Stubbs (2008) señala que es relevante indicar que el vocablo "inclusión” hace referencia a la edad, género, grupo étnico, lenguaje, estado de salud y económico, religión, estilo de vida y no solo a una discapacidad notoria o aparente; desde este punto de vista, la educación inclusiva universitaria, no puede concebirse como aquella que forma a sus estudiantes desde un modelo homogéneo, la universidad está llamada a atender en forma diversificada a sus estudiantes, para que realmente reciba el calificativo de incluyente.

Según la Asociación Nacional de Universidades e Instituciones de Educación Superior ANUIES (2018), en el año de 2018 en México, existían alrededor de 4,210,250 estudiantes de nivel superior, donde el $49.7 \%$ eran hombres y el 50.3\% eran mujeres. En sus estadísticas, la ANUIES indica que, del total de alumnos matriculados, el 1\% representaba a alumnos con alguna discapacidad, sin embargo, no hace referencia a las necesidades específicas de cada uno de ellos según su contexto cultural o social. Una característica relevante de estos resultados pone en evidencia que aquellos estudiantes que presentan alguna condición o necesidad educativa no se reportan y por lo tanto es muy probable que se encuentren excluidos de la asistencia especial con estrategias adecuadas para ellos.

En el año 2017, un artículo de Cruz y Casillas denominado: las instituciones de educación superior y los estudiantes con discapacidad en México, expone un estudio donde se revisan alrededor de 53 universidades mexicanas, dentro de las cuales, solo 12 contaban hasta ese momento con acciones o programas de apoyo a alumnos con discapacidad, según el punto de vista de los autores señalan que "en su mayoría son solo iniciativas de inclusión". En el estudio realizado, señalan que existen instituciones que adoptan programas específicos de 
atención a alumnos con discapacidad, siendo el único criterio de inclusión; por ejemplo: Universidad Nacional Autónoma de México (UNAM), Universidad Veracruzana (UV), Universidad Autónoma de Tlaxcala (UATX), Universidad Autónoma de San Luis Potosí (UASLP), la Universidad Autónoma de Nuevo León (UANL) y la Universidad Juárez del Estado de Durango (UJED). En conjunto, estos estudios proporcionan importantes resultados sobre la situación que guarda la inclusión en el nivel superior, sobre la base de lo anterior, es muy significativo observar que el único criterio de inclusión está orientado hacia alumnos con discapacidad, lo cual implica que no quedan visibilizados los estudiantes con necesidades educativas, lo cual puede determinar una condición de riesgo y vulnerabilidad ante las circunstancias que deben afrontar en su formación universitaria.

De acuerdo con la Declaración de Yucatán (2008) la cual fue escrita en correspondencia con la Convención sobre los Derechos de las Personas con Discapacidad, establece que es "necesario reconocer la universalidad, indivisibilidad, progresividad, interdependencia, e interrelación de todos los derechos humanos y libertades fundamentales, así como la necesidad de garantizar que las personas con discapacidad en las universidades, los ejerzan plenamente y sin discriminación" (p. 1), a este efecto, se considera fundamental que las universidades garanticen a sus estudiantes con discapacidad, que el tránsito por los espacios de su formación profesional, se realizará con el pleno goce de sus derechos humanos y sin discriminación, sin embargo, previamente se ha demostrado que a pesar de que este acuerdo se realizó hace más 10 años, aún falta mucho camino por recorrer para el pleno cumplimiento del mismo.

Desde este punto de vista, los resultados de la Encuesta Nacional de Discriminación 2017 (ENADIS) demuestra claramente que una de las principales problemáticas de las personas con discapacidad son la falta de oportunidades para encontrar empleo. Entre este grupo de población, el $48.1 \%$ percibe que sus derechos le son respetados poco o nada. Al $15.1 \%$ de quienes tienen 18 años y más y viven con alguna discapacidad, le fueron negadas becas u otros programas sociales del gobierno; al 6.5\% le fue negada la oportunidad de seguir estudiado. De acuerdo con estos resultados, la universidad tiene un reto muy grande, el cual implica considerar la construcción de propuestas que comprometan su completa operación. Aun cuando se están realizando esfuerzos, existe, no obstante, una distancia sustancial entre la asistencia que reciben los alumnos con discapacidad en el nivel de educación básica y la educación superior.

Las universidades aún tienen dificultades para implementar un modelo educativo incluyente; de conformidad con resultados de investigaciones recientes, se advierten elementos de resistencia al cambio, con barreras importantes para la plena inclusión, Tapia y Manosalva (2012) puntualizan en sus investigaciones, que a medida que se avanza en los niveles del 
sistema educativo, las barreras se hacen muy complejas y es el nivel de educación superior el que muestra mayores resistencias y barreras dentro del proceso de integración. Torres (2011), subraya que la inclusión educativa puede y debe ser considerada una actitud, en este sentido, se convierte en un sistema de creencias y valores que estará presente en la toma de decisiones de aquellos que apuestan por ella. Con el objetivo de estudiar los enfoques inclusivos que se demandan en la educación superior, se plantea el desarrollo de una dimensión que se afianza en el diseño y desarrollo de estrategias psicopedagógicas que permitan dar respuesta a la diversidad y con ello crear ambientes educativos incluyentes.

Por lo expuesto anteriormente, es necesario que, desde la dimensión educativa, se acepte una mayor responsabilidad en la creación de procesos innovadores que garanticen a las personas con discapacidad el acceso al sistema formativo. En consecuencia, las instituciones de educación superior deben desarrollar, dentro de sus políticas educativas, prácticas inclusivas mediante la capacitación de sus docentes, que les permitan desarrollar pedagogías flexibles que integren a la diversidad del estudiantado.

En la actualidad, se desarrolla un creciente interés por la inclusión de las personas con discapacidad, sin duda alguna, esto se debe a que conforman un grupo reducido y segregado en diferentes dimensiones del contexto social, los recientes desarrollos en el campo de la educación inclusiva han puesto de manifiesto que aun cuando existen normativas y políticas nacionales sobre la discapacidad, tal es el caso del acuerdo de Yucatán, esta población requiere de condiciones que favorezcan su participación y aprendizaje, que se busque reducir las barreras que los mantienen excluidos; en atención a que la carencia de relaciones igualitarias ha permanecido representando un obstáculo pendiente de vencer.

De acuerdo con las estadísticas que emitió la Organización Mundial de la Salud en el 2011, se reportan los peores resultados de las personas con discapacidad en términos de salud, de contexto académico, de menor participación económica y de tasas de pobreza, en clara desigualdad con las personas sin discapacidad. Todo ello responde a los obstáculos que limitan su acceso a los servicios considerados básicos, particularmente la salud, educación, empleo, entre otros (OMS, 2011), en este mismo sentido, Boudeguer y Squella (2010) señalan que la discapacidad la generan barreras físicas del entorno, aludiendo al enfoque actual de accesibilidad, transformándose en aquella: "capacidad de aproximarse, acceder, usar y salir de todo espacio o recinto con independencia, facilidad y sin interrupciones" por lo tanto, el medio físico influye en la manifestación o la eliminación de la discapacidad. De acuerdo con Raphael (2012b), argumenta que nuestra sociedad mexicana sigue siendo fuertemente discriminatoria, lo cual nos aleja de la democracia.

RIAEE - Revista Ibero-Americana de Estudos em Educação, Araraquara, v. 15, n. esp. 4, p. 2713-2726, dez., 2020. e-ISSN: 1982-5587 
En relación con las barreras que imposibilitan la inclusión plena de las personas con discapacidad en la educación superior, López (2014) concluye en sus investigaciones que:

[...]la política educativa y las prácticas escolares y pedagógicas crean barreras para la inclusión, en la medida en que tienden a la individualización, a la segregación y a la desresponsabilización del aprendizaje y la participación de los estudiantes con necesidades educativas especiales (p. 279).

Diversas investigaciones han revelado hasta el momento, que no hay una clara evidencia de que la inclusión se haya consolidado en la educación superior, indican que aún existe una distancia sustancial entre la asistencia y apoyo que se les da a los alumnos con discapacidad en el nivel de educación básica y en niveles educativos superiores. Aunque se han hecho progresos considerables en este rubro, muchas cuestiones importantes permanecen todavía sin resolver, de acuerdo con los análisis presentados por Alcántara y Navarrete (2014) y Cruz y Casillas (2017) señalan que las instituciones de educación superior, no solamente en México, están respondiendo con dilación al implantar un modelo de educación inclusiva, por lo tanto, es fundamental que desde el contexto escolar, se asuma un compromiso relevante en la creación de metodologías innovadoras que garanticen a las personas con discapacidad la plena inclusión al sistema educativo.

\section{Docencia y educación inclusiva universitaria}

Las instituciones de educación superior deben implementar, dentro de sus políticas educativas, prácticas inclusivas mediante la capacitación de sus docentes, que les permitan desarrollar estrategias de enseñanza-aprendizaje-evaluación con enfoque inclusivo, de tal manera que se promueva la incorporación de las personas con discapacidad.

Es esencial, revisar las propuestas que, fundamentadas en investigaciones previas, se han realizado sobre la importancia de la capacitación docente. De acuerdo con este aspecto, Yupanqui, Aranda y Vásquez (2014) señalan que las intervenciones de los docentes deben orientarse hacia el fomento de la educación sobre el uso del lenguaje inclusivo, el apoyo en la identificación oportuna de condiciones de riesgo y el diseño e implementación de las adecuaciones curriculares para el manejo de conductas disruptivas; las adaptaciones ambientales y de accesibilidad, así como la educación en técnicas de integración sensorial entre otras.

En la opinión de Rojas (2011), un docente debe de atender las necesidades sociales de la comunidad, es decir, buscar hacer un bien a la sociedad en la que se desarrolla, sin dejar de 
lado su crecimiento personal y profesional. Desde esta perspectiva, una educación inclusiva apunta a la construcción de un vínculo entre el docente y cada uno de sus estudiantes basado en el pleno reconocimiento y el respeto mutuo (LÓPEZ, 2016). Una parte integral de la formación docente implica acceder a prácticas especializadas en temas de atención a la diversidad, en ese marco, implica la capacitación en diversas dimensiones, desde lo disciplinar, pedagógico, desarrollo humano y otros.

Por otra parte, Coll (1996) señala que la actividad del profesorado tiene una estrecha relación con la manera cómo aprende y se desarrolla el estudiantado, con las dificultades y problemas que se encuentran cuando lleva a cabo nuevos aprendizajes y con las intervenciones dirigidas a apoyarle para que supere dichas dificultades. En este contexto, el docente debe responder a las nuevas exigencias que le plantea la inclusión en el aula, superar sus propias barreras, para diseñar ambientes de aprendizaje que favorezcan la reducción de barreras para el aprendizaje y la participación de sus alumnos.

Por otra parte, Fernández (2012) destaca que el personal docente participa en el análisis, planificación, desarrollo y la modificación de los procesos educativos; por tanto, deben formarse para desarrollar una serie de competencias que le permitan ser inclusivo y contribuir en su práctica profesional. Así mismo, Alcaín y Medina (2017) comentan que la labor docente es la principal vía de las estrategias de educación con inclusión en las universidades, señalan que es necesario que se revalore la función del catedrático dentro del aula, que deje de percibirse la atención a la diversidad, desde un concepto de carga extra; sino transformarla en una oportunidad de crecimiento profesional. El docente desde su función intermediadora debe considerar en la planificación sistemática de contenidos, las diferencias de mediación educativa, con el fin de lograr los objetivos esperados, considerando la implementación de la evaluación formativa para el aprendizaje y no sólo del aprendizaje, la cual permitirá la correcta toma de decisiones en beneficio de la mejora de los aprendizajes, respondiendo así a las exigencias que plantea la educación inclusiva. El docente y las instituciones tienen que estar en capacitación permanente, con la finalidad de desarrollar las habilidades necesarias que le permitan llevar a cabo estas metas.

Otro elemento primordial que se destaca es la construcción de la dinámica en el aula, es la creación de espacios de respeto e incluyentes, en los cuales no se dé cabida a actos de discriminación; a este respecto, se hace necesario generar espacios de sensibilización y toma de conciencia frente a la diversidad educativa en las universidades, sobre esta base, cabe reflexionar que el proceso de inclusión es un tema emergente, que demanda acciones globales de la sociedad en general. Finalmente, Almanzor (2002) considera que la capacitación 
pedagógica del docente puede contribuir a que los profesores reflexionen acerca de su inserción en el tiempo que les ha tocado vivir y que participen de una manera más protagónica en la construcción de un porvenir mejor. Las investigaciones hasta ahora presentadas, proporcionan evidencia de que la formación docente es un rubro fundamental en el logro de los fines que persigue la educación inclusiva, el docente debe autoevaluarse en su práctica, identificar sus limitaciones y barreras con el propósito de alcanzar un cambio de paradigma, reconfigurar su función de mediador y facilitador de espacios significativamente incluyentes, por medio de la aplicación de estrategias diferenciadas dirigidas a la atención adecuada de la diversidad.

\section{Estrategias pedagógicas para la educación inclusiva}

Las estrategias pedagógicas para la diversidad permiten abatir las rutinas igualitarias y homogeneizadoras, se orientan hacia el diseño de metodologías que operen desde una visión innovadora y creativa los recursos disponibles, tales es el caso de los materiales de apoyo y didácticos, programas de orientación inclusiva, organización de tiempos y espacios, reestructura de formas de interacción y movilización del grupo.

Por otra parte, De la Oliva (2015), propone la operación de los proyectos formativos, argumenta, que el uso de estas estrategias favorece la inclusión, llevan a la superación de las posibles barreras que existan en el aula. De acuerdo con la Ley General para la Inclusión de las Personas con Discapacidad, (2018) se destaca la importancia de la función docente, advirtiendo algunas características fundamentales de esta labor, en el apartado VI señala que el docente debe proporcionar a los estudiantes con discapacidad materiales y ayudas técnicas que apoyen su rendimiento académico, también se realizan señalamientos orientados a los directivos, los cuales deben procurar y equipar los planteles y centros educativos con libros en braille, materiales didácticos, apoyo de intérpretes de lengua de señas mexicana o especialistas en sistema braille, equipos computarizados con tecnología destinada a personas invidentes, todos aquellos apoyos que se identifiquen necesarios para brindar una educación con calidad, en el apartado VII, se establece la importancia de incluir la enseñanza del Sistema de Escritura Braille y la Lengua de Señas Mexicana en la educación pública y privada, fomentando la producción y distribución de libros de texto gratuitos en Sistema de Escritura Braille, macrotipos y textos audibles que complementen los conocimientos de los alumnos con discapacidad y finalmente en el apartado XIII, se advierte la importancia de impulsar a los estudiantes que necesitan cumplir con el requisito del servicio social para que ofrezcan su apoyo a las personas con discapacidad. 
De acuerdo con Ortiz (2000), el primer paso hacia la escuela inclusiva es la aceptación incondicional de las diferencias, debido a que la diversidad fortalece al alumnado, al ofrecerle alternativas de aprendizaje. En efecto, en la comprensión de la enseñanza, es donde se percibe lo complejo de la acción docente, por lo que para garantizar lo anterior, este autor sugiere adoptar una propuesta interactiva de enseñanza-aprendizaje. Estos preceptos están relacionados con la calidad de la educación, por lo cual es esencial que las instituciones de educación superior garanticen y generen constantemente apoyos y recursos orientados a la consolidación de procesos inclusivos.

En el mismo orden de ideas, se plantea una estrategia para la práctica docente sustentada en el diseño universal para el aprendizaje (DUA), dado que permite el desarrollo de oportunidades e interacciones para el aprecio de la diversidad y ofrece avances en la estructura de acceso e innovación en la metodología de la enseñanza y aprendizaje. En dicha construcción, tanto estudiantes y docentes desempeñan el rol de mediadores y pueden proveer de andamios o ayudas ajustadas a las necesidades de otros estudiantes. Dado que mediante la inclusión educativa se pretende generar espacios abiertos a la diversidad y potenciar las capacidades; el énfasis en la interacción y en el intercambio de experiencias desde diferentes miradas, resulta fundamental para la generación de saberes compartidos.

En vista de todo lo que se ha dicho hasta ahora, se pone de relieve el gran reto que tiene la educación superior, en donde las políticas públicas del Estado mexicano representan un rol fundamental, las cuales deberán configurarse esencialmente con enfoque de igualdad y no discriminación, en México, se ha dado un paso sustancial, en 2019 se ha modificado el artículo $3^{\circ}$ de la Constitución Política de los Estados Unidos Mexicanos (Última modificación 9 de agosto de 2019), los cambios se señalan a la fracción II, correspondiente a los criterios que orientarán a la educación, quedando enmarcado que la educación:

“e) Será equitativo, para lo cual el Estado implementará medidas que favorezcan el ejercicio pleno del derecho a la educación de las personas y combatan las desigualdades socioeconómicas, regionales y de género en el acceso, tránsito y permanencia en los servicios educativos... Asimismo, se respaldará a estudiantes en vulnerabilidad social, mediante el establecimiento de políticas incluyentes y transversales. f) Será inclusivo, al tomar en cuenta las diversas capacidades, circunstancias y necesidades de los educandos. Con base en el principio de accesibilidad se realizarán ajustes razonables y se implementarán medidas específicas con el objetivo de eliminar las barreras para el aprendizaje y la participación; g) Será intercultural, al promover la convivencia armónica entre personas y comunidades para el respeto y reconocimiento de sus diferencias y derechos, en un marco de inclusión social; h) Será integral, educará para la vida, con el objeto de desarrollar en las personas capacidades cognitivas, socioemocionales y físicas que les permitan

RIAEE - Revista Ibero-Americana de Estudos em Educação, Araraquara, v. 15, n. esp. 4, p. 2713-2726, dez., 2020. e-ISSN: 1982-5587 
alcanzar su bienestar, i) Será de excelencia, entendida como el mejoramiento integral constante que promueve el máximo logro de aprendizaje de los educandos, para el desarrollo de su pensamiento crítico y el fortalecimiento de los lazos entre escuela y comunidad" (2019, p.7).

En conjunto, estas modificaciones al artículo 3ro., implican el diseño de una estructura que configure los procesos que posibilitarán su cumplimiento e implementación, por lo tanto, será necesario articular esfuerzos al interior del tejido social, además del desarrollo de metodologías de enseñanza sustentadas en el conocimiento y en la experiencia, entre muchas otras iniciativas. Por lo tanto, resulta necesario llevar a cabo reformas educativas en la formación docente de todos los niveles educativos, con el fin de dotar a los maestros de las competencias necesarias que permitan enfrentar los desafíos de la educación inclusiva y acercar su labor a las necesidades y expectativas de los estudiantes, quienes viven en un mundo de cambios permanentes.

Finalmente, cabe mencionar que el término de educación inclusiva aglutina una multitud de procesos que implican a la sociedad en su conjunto; desde la visión de Díaz, Zárate y Ortiz (2017) la educación inclusiva es una aspiración y una necesidad de la universidad actual, que debe tener fundamentalmente el principio básico de la implicación de todos y la aceptación mutua; en este sentido, lo que no se cumple espontáneamente, se enseña y se aprende, y forma parte de la vida. En una sociedad, comunidad, escuela, grupo y en un sujeto, debe existir la capacidad para la aceptación, la convivencia, la ayuda, la tolerancia y el espíritu de superación. Desde este contexto, es fundamental poner en marcha estrategias dirigidas a reducir, minimizar o eliminar las barreras que obstaculizan la presencia, participación y aprendizaje en la educación superior, sobre todo, las estrategias que consideren propuestas de leyes o normas, la optimización de la infraestructura física con el respaldo de las tecnologías de información y comunicación, así mismo, aquellas que promuevan el desarrollo de programas específicos para la inclusión y la capacitación continua de la comunidad universitaria en los ámbitos implicados en la educación inclusiva.

Para concluir, cabe mencionar lo que expone Jennifer O’Donoghue (2019), en relación con el concepto de educación inclusiva del cual afirma que debe ser suficientemente amplio para poder responder y promover la diversidad de cualquier índole para que todas y todos puedan estar, aprender y participar en la escuela. La educación incluyente no es una batalla ganada; es un proceso continuo de identificar y eliminar las actitudes, prioridades y paradigmas que nos han impedido avanzar. 


\section{REFERENCIAS}

AINSCOW, M.; MILES, S. Por una educación para todos que sea inclusiva: ¿Hacia dónde vamos ahora? Perspectivas, v. 38, n. 1, p. 17-44, 2008. Disponible en:

http://www.ibe.unesco.org/fileadmin/user_upload/Publications/Prospects/Prospects145_spa.p df\#page=20. Acceso en: 10 sep. 2020.

AINSCOW, M.; BOOTH, T.; DYSON, A. Improving schools, developing inclusion. London: Routledge Taylor \& Francis Group, 2006.

ALCAÍN M. E.; MEDINA G. M. Hacia una educación universitaria inclusiva: realidad y retos. Revista Digital de Investigación en Docencia Universitaria, v. 11, n. 1, p. 4-19, 2017. DOI: https://doi.org/10.19083/ridu.11.530

ALMANZOR, F.C. Importancia de la capacitación pedagógica del docente. In: Análisis documental del proceso de inclusión en la educación. Disponible en:

http://www.gdl.uag.mx/63/a04-02.htm. Acceso en: 10 sep. 2020.

ANUIES. Visión y acción 2030. Propuesta de la ANUIES para renovar la educación superior en México. 2018. Disponible en:

https://visionyaccion2030.anuies.mx/Vision_accion2030.pdf. Acceso en: 10 sep. 2020.

BOUDEGUER, A; PRETT, P.; SQUELLA, P. Manual de accesiblidad universal: Ciudad y espacio para todos. Santiago de Chile: Corporación Ciudad Accesible, 2010.

COLL, C. Psicología y curriculum. Una aproximación psicopedagógica a la elaboración del curriculum escolar. Barcelona: Paidós, 1991.

COLL, C. Psicopedagogía: confluencia disciplinar y espacio profesional, 1996.

MEXICO. Constitución Política de los Estados Unidos Mexicanos. Diario Oficial de la Federación el 5 feb. 1917.Última modificación 9 de agosto de 2019, Disponible en: https://www.gob.mx/indesol/documentos/constitucion-politica-de-los-estados-unidosmexicanos-97187. Acceso en: 10 sep. 2020.

CRUZ, V. R.; CASILLAS, A. M. A. Las instituciones de educación superior y los estudiantes con discapacidad en México. Revista de educación superior, v. 46, n. 181, p. 37-53, 2017. DOI: https://doi.org/10.1016/j. resu.2016.11.002

DE LA CRUZ, G. Inclusión en educación superior: de la atención a la diversidad al facultamiento del estudiantado. España. Revista científica electrónica de Educación y Comunicación en la Sociedad del Conocimiento, Año XII, v. II n. 12 jul./dic. 2012. ISSN: 1695-324X.

DE LA OLIVA, D.; TOBÓN, S.; PÉREZ, S.; KARINA, A.; ROMERO, J. El Proceso de Inclusión Social desde la Socioformación: Análisis de Concepciones sobre Discapacidad y Necesidades Educativas Especiales. Paradigma, v. 36, p. 2, 2015. Disponible en: http://www.scielo.org.ve/pdf/pdg/v36n2/art04.pdf. Acceso en: 10 sep. 2020. 
DECLARACIÓN DE YUCATÁN. 2008. Disponible en: http://www.ddu.unam.mx/DDU/ Documentos/Ponencias II sesion/DECLARACION YUCATAN.pdf. Acceso en: 10 sep. 2020.

DELGADO, W. Inclusión: principio de calidad educativa desde la perspectiva del desarrollo humano. Revista Educación, Costa Rica, v. 31, n. 2, p. 45-58, 2007.

DÍAZ, O.; ZÁRATE, R.; ORTIZ, G. L. Educación superior inclusiva: un reto para las prácticas pedagógicas. Revista Electrónica Educare, v. 21, n. 3, p. 1-24, 2017, sep./dic. 2017. DOI: http://dx.doi.org/10.15359/ree.21-3.15

FERNÁNDEZ, F. Una mirada a los desafíos de la educación superior en México. Innovación Educativa, v. 17, n. 74, p. 183-207, 2017.

INEGI. Instituto Nacional de Estadística y Geografía. Encuesta nacional sobre discriminación en México (ENADIS). Ciudad de México: INEGI, 2017.

JIMÉNEZ, R. Las personas con discapacidad en la educación superior. Una propuesta para la diversidad e igualdad. Costa Rica: Fundación Justicia y Género San José, 2002. p. 138. MEXICO. Ley General para la Inclusión de las Personas con Discapacidad. Diario Oficial de la Federación. México, 12 jul. 2018. Disponible en:

http://www.diputados.gob.mx/LeyesBiblio/pdf/LGIPD_120718.pdf. Acceso en: 10 sep. 2020.

MONEREO, I. S. (Coords.). El asesoramiento psicopedagógico: una perspectiva profesional y constructivista. Psicología y Educación. Madrid: Alianza Editorial. p. 33-52.

MORENO, M. Integración/inclusión de las personas con discapacidad en la Educación Superior, en Informe sobre la Educación Superior en América Latina y el Caribe 20002005. La metamorfosis de la Educación Superior. Caracas: IESALC/ UNESCO, 2006.

OCAÑA, S.; HERNÁNDEZ, L.; SÁNCHEZ, M.; LINARES, M.; GUERRA, H.; REYES, M.; SOTO, O. Estrategias de inclusión en la educación universitaria en el área de ciencias: retos bioéticos de la actualidad. Revista Iberoamericana de Bioética, n. 10, p. 1-13, [2019].

O’DONOGHUE, J. Una educación incluyente desde la Constitución. Educación Futura. 16 ene. 2019 Disponible en: http://www.educacionfutura.org/una-educacion-incluyente-desde-laconstitucion/. Acceso en: 10 sep. 2020.

Organización Mundial de la Salud. Informe mundial sobre la discapacidad. Ginebra: OMS, 2011.

ORTIZ, M. Hacia una educación inclusiva. La educación especial ayer, hoy y mañana.

Revista Siglo Cero, v. 31, n. 187, p. 5-11, 2000.

ROJAS ATRAVIA, C. E. Ética profesional docente: un compromiso pedagógico humanístico. Revista humanidades, n. 1, p. 1-22, 2011.

SALINAS ALARCÓN, M. L. La inclusión en la educación superior desde la voz de estudiantes chilenos con discapacidad. Revista Ibero-Americana de Educação, v. 63, p. 77 98, 2013. 
SEP. Modelo Educativo. México, 2016. Disponible en:

https://www.gob.mx/cms/uploads/attachment/file/114501/Modelo_Educativo_2016.pdf.

Acceso en: 10 sep. 2020.

TOBÓN, S. El enfoque socioformativo y las competencias: ejes claves para transformar la educación. In: TOBÓN, S.; JAIK DIPP, A. (Coords.). Experiencias de Aplicación de las competencias en la educación y el mundo organizacional. Durango, México: ReDIE, 2012. Disponible en: http://iunaes.mx/wp-content/uploads/2013/04/Experiencias-deAplicacion.pdffpage=10. Acceso en: 10 sep. 2020.

TORRES-GONZÁLEZ, J. Psique: La inclusión educativa, la necesidad de transformación ante nuevas realidades. Colombia. Boletín Científico Sapiens Research, v. 1.1, p. 11-15, 2011.

\section{YUPANQUI CONCHA, A.; ARANDA FARÍAS, C. A.; VÁSQUEZ OYARZUN, C.}

Educación inclusiva y discapacidad: su incorporación en la formación profesional de la educación superior. Revista de la Educación Superior, v. 3, n. 171, p. 93-115, jul./sep. 2014. ISSN: 0185-2760.

\section{Cómo referenciar este artículo}

JÁCOME, G. E. L.; ÁLVAREZ, M. C. M. La educación inclusiva en el marco de la educación superior. Revista Ibero-Americana de Estudos em Educação, Araraquara, v. 15, n. esp. 4, p. 2713-2726, $\quad$ dez., $2020 . \quad$ e-ISSN: 1982-5587. DOI: https://doi.org/10.21723/riaee.v15iesp4.14519

Remitido el: 10/09/2019

Revisiones requeridas el: 10/01/2020

Aprobado el: $30 / 04 / 2020$

Publicado el: $01 / 12 / 2020$ 